\title{
TWO-POINT PROJECTION ESTIMATES FOR HARMONIC MEASURE
}

\author{
DIMITRIOS BETSAKOS
}

\begin{abstract}
A theorem of Beurling and Nevanlinna gives a lower bound for the harmonic measure of a compact set in the unit disk in terms of the harmonic measure of a projection of the set. We prove a generalization of that result involving the sum of harmonic measures evaluated at two points.
\end{abstract}

\section{INTRODUCTION}

Let $D$ be a domain in the complex plane $\mathbb{C}$ and let $E$ be a Borel set on $\partial D$. The harmonic measure of $E$ relative to $D$ is the function $\mathrm{u}$ which is harmonic in $D$ and has boundary values $u=1$ on $E$ and $u=0$ on $\partial D \backslash E$ (in the sense of the generalized Dirichlet problem; see e.g. [6, Chapter 4]. For a compact set $K \subset \mathbb{C}$, let $\omega(z, K, D)$ denote the harmonic measure of the set $K \cap \partial(D \backslash K)$, relative to $D \backslash K$, evaluated at $z \in D \backslash K$; we also set $\omega(z, K, D)=1$ for $z \in K$. For the harmonic measure $\omega(z, K, \mathbb{D})$, where $\mathbb{D}$ is the unit disk, we will use the simpler notation $\omega(z, K)$. Let also $g(\cdot, \zeta)$ denote the Green's function of $\mathbb{D}$ with pole at $\zeta \in \mathbb{D}$.

1991 Mathematics Subject Classification. Primary: 30C85, 31A15.

Key words and phrases. Harmonic measure, Green's function. 
A classical theorem of A.Beurling and R.Nevanlinna (see e.g. [6, §4.5]) asserts that for all $z \in \mathbb{D}$,

$$
\omega(z, K) \geq \omega\left(-|z|, K^{*}\right)
$$

Here and below $K^{*}=\{|z|: z \in K\}$ (the circular projection of $K$ onto the radius $(0,1]$ of $\mathbb{D})$.

We will prove the following generalization of (1.1).

Theorem 1.1. Let $0 \leq r<1, \pi / 2 \leq \theta \leq \pi$, and let $K$ be a compact set in the closure $\overline{\mathbb{D}}$ of the unit disk. Then

$$
\omega\left(r e^{i \theta}, K\right)+\omega\left(r e^{-i \theta}, K\right) \geq \omega\left(r e^{i \theta}, K^{*}\right)+\omega\left(r e^{-i \theta}, K^{*}\right)
$$

This is only slightly stronger than a result in [1] (in [1] we considered only the case $\theta=\pi / 2$ ) but the proof we give in the present paper is different. It combines a method of A.Baernstein [3, Lemma 2] with a method of B.Øksendal [4]. The method used by Beurling and Nevanlinna for the proof of (1.1) involves a circular projection of the Riesz measure of a certain superharmonic function. We note that a direct application of that method cannot prove the theorem. Instead, we do the projection of $K$ step by step (as in [4]) and in each step we apply a transport of the Riesz measure (as in [3]).

The Beurling-Nevanlinna projection theorem involves the circular projection of both the set $K$ (onto the positive real axis) and of the base point $z$ (onto the negative real axis). Our next result also involves such a double projection but now we have two base points. 
Theorem 1.2. Let $0 \leq r<1,0<\theta<\pi / 2$, and let $K$ be a compact set in $\overline{\mathbb{D}} \cap\{z: \Re z \geq 0\}$. Then

$$
\omega\left(r e^{i \theta}, K\right)+\omega\left(r e^{-i \theta}, K\right) \geq \omega\left(i r, K^{*}\right)+\omega\left(-i r, K^{*}\right) .
$$

It is easy to see that the assumption $K \subset\{z: \Re z \geq 0\}$ cannot be omitted.

Several results, open problems, and references related to Theorems 1.1 and 1.2 appear in [2].

\section{Proofs}

We first state a number of lemmas for reference later.

Lemma 2.1. Let $D_{1} \subset D$ be two domains in the plane and $K$ be a compact set in $\overline{D \backslash D_{1}}$. Then for $z \in D_{1}$

$$
\omega(z, K, D)=\int_{\partial D_{1} \cap D} \omega(\zeta, K, D) \omega\left(z, d \zeta, D_{1}\right) .
$$

The lemma follows at once from the strong Markov property of Brownian motion; see e.g. [5, p.88]. Alternatively, (2.1) follows from the maximum principle applied to the domain $D_{1}$.

Lemma 2.2. Let $0<\alpha<\beta<\pi, 0 \leq r, s<1$. Then

$$
g\left(r, s e^{i \alpha}\right)>g\left(r, s e^{i \beta}\right) .
$$


This lemma can be proved easily using the explicit expression for the Green's function $[6, \S 4.4]$ :

$$
g(z, \zeta)=\log \frac{|1-z \bar{\zeta}|}{|z-\zeta|}=\log \left(1+\frac{\left(1-|z|^{2}\right)\left(1-|\zeta|^{2}\right)}{|z-\zeta|^{2}}\right)
$$

Lemma 2.3. Let $0<r<1,0<s<1$. The function $f$ with

$$
f(\theta)=g\left(i r, s e^{i \theta}\right)+g\left(-i r, s e^{i \theta}\right), \theta \in[0, \pi / 2],
$$

is strictly increasing.

Proof. Because of (2.3) it suffices to prove that $h^{\prime}(\theta)>0$, where

$$
h(\theta)=\left(1+\frac{\left(1-r^{2}\right)\left(1-s^{2}\right)}{r^{2}+s^{2}-2 r s \sin \theta}\right)\left(1+\frac{\left(1-r^{2}\right)\left(1-s^{2}\right)}{r^{2}+s^{2}+2 r s \sin \theta}\right) .
$$

Simple calculations show that

$$
h^{\prime}(\theta)=\frac{4 r^{2}\left(1-r^{4}\right)\left(1-s^{4}\right) \sin 2 \theta}{\left(r^{2}+s^{2}-2 r \sin \theta\right)^{2}\left(r^{2}+s^{2}+2 r \sin \theta\right)^{2}}
$$

which is positive for $\theta \in(0, \pi / 2)$.

Before stating the next lemma we need to define a geometric transformation in the plane (see [4, p.192], [3, p.222]): Let $\varphi \in[0,2 \pi)$ and $l(\varphi)$ be the oriented line passing through the points 0 and $e^{i \varphi}$ with orientation from 0 to $e^{i \varphi}$. Let $H$ (or $H_{\varphi}$ ) be the half-plane bounded by $l(\varphi)$ and containing the point $e^{i(\varphi-\pi / 2)}$. For $z \in \mathbb{C}$ and $A \subset \mathbb{C}$, let $\mathcal{R} z$ and $\mathcal{R} A$ denote the reflection of $z$ and $A$ in $l(\varphi)$ (we use the notation $\mathcal{R}_{\varphi}$ if we want to stress the dependence on $\varphi$ ). If $z \in \mathbb{C}$, we denote by $\tilde{z}$ the unique point that belongs to the set $H \cap\{z, \mathcal{R} z\}$. For $A \subset \mathbb{C}$ we let $\tilde{A}_{\varphi}=\{\tilde{z}: z \in A\}$. 
The following lemma was proved in [4], [3]. We state it here in a different form:

Lemma 2.4. Let $K$ be a compact subset of $\overline{\mathbb{D}}, z \in \mathbb{D}$, and $\varphi \in[0,2 \pi)$. Then

$$
\begin{aligned}
\omega(z, K) & \geq \omega\left(\mathcal{R} z, \tilde{K}_{\varphi}\right) \\
\omega(z, K)+\omega(\mathcal{R} z, K) & \geq \omega\left(z, \tilde{K}_{\varphi}\right)+\omega\left(\mathcal{R} z, \tilde{K}_{\varphi}\right) .
\end{aligned}
$$

We extend Lemma 2.4 as follows:

Lemma 2.5. Let $0 \leq r<1$ and $\varphi \in(0, \pi / 4]$. Also let $K$ be a compact set in $\overline{\mathbb{D}} \cap\{z: \Re z \geq 0, \Im z \geq 0\}$. Then

$$
\omega(i r, K)+\omega(-i r, K) \geq \omega\left(i r, \tilde{K}_{\varphi}\right)+\omega\left(-i r, \tilde{K}_{\varphi}\right) .
$$

Proof. (cf. [3, Lemma 2]) By an approximation and a majorization argument we may assume that $K \cap l(\varphi)=\emptyset, K \subset \mathbb{D}$, and that

$$
(K \cap H) \cap \mathcal{R}(K \cap \mathcal{R} H)=\emptyset .
$$

Let

$$
u(z)= \begin{cases}\omega(z, K), & z \in \mathbb{D} \backslash K, \\ 1, & z \in K .\end{cases}
$$

Then $u$ is a bounded superharmonic function in $\mathbb{D}$ and $u=0$ on $\partial \mathbb{D}$. Therefore, by the Riesz decomposition theorem, there exists a positive measure $\mu$ (the Riesz measure of $u$ ) such that

$$
u(z)=\int_{K} g(z, \zeta) d \mu(\zeta)
$$

We define a measure $\nu$ on $\tilde{K}_{\varphi}$ as follows: For $X \subset \tilde{K}_{\varphi}$, we write $X=A \cup B$ with $A \subset K \cap H$ and $B \subset \mathcal{R}(K \cap \mathcal{R} H)$, and set $\nu(X)=$ 
$\mu(A)+\mu(\mathcal{R} B)$. We also define

$$
v(z)=\int_{\tilde{K}_{\varphi}} g(z, \zeta) d \nu(\zeta) .
$$

Proposition 1: $v(i r)+v(-i r) \leq u(i r)+u(-i r)$.

Proposition 2: $v(z) \geq 1$, for $z \in \tilde{K}_{\varphi}$.

Suppose for the moment that Propositions 1 and 2 are true. Proposition 2 and the maximum principle imply that $v(z) \geq \omega\left(z, \tilde{K}_{\varphi}\right), z \in$ $\mathbb{D} \backslash \tilde{K}_{\varphi}$, and, in particular, that

$$
v(i r)+v(-i r) \geq \omega\left(i r, \tilde{K}_{\varphi}\right)+\omega\left(-i r, \tilde{K}_{\varphi}\right) .
$$

Now (2.13) and Proposition 1 imply (2.9). So it remains to prove the propositions. For the proof of both propositions we will need the following formula which comes from the construction of the measure $\nu$ and a change of variable:

$$
\begin{aligned}
v(z)=\int_{\tilde{K}_{\varphi}} g(z, \zeta) d \nu(\zeta) & =\int_{K \cap H} g(z, \zeta) d \nu(\zeta)+\int_{\mathcal{R}(K \cap \mathcal{R} H)} g(z, \zeta) d \nu(\zeta) \\
& =\int_{K \cap H} g(z, \zeta) d \mu(\zeta)+\int_{K \cap \mathcal{R} H} g(z, \mathcal{R} \zeta) d \mu(\zeta)
\end{aligned}
$$

Proof of Proposition 1: By (2.14)

$$
\begin{aligned}
v(i r)+v(-i r) & =\int_{K \cap H}[g(i r, \zeta)+g(-i r, \zeta)] d \mu(\zeta)+ \\
& +\int_{K \cap \mathcal{R} H}[g(i r, \mathcal{R} \zeta)+g(-i r, \mathcal{R} \zeta)] d \mu(\zeta)
\end{aligned}
$$

For $\zeta \in K \cap \mathcal{R} H$ we have

$$
0<\arg \mathcal{R} \zeta<\arg \zeta<\frac{\pi}{2} .
$$


Hence Lemma 2.3 yields

$$
g(i r, \mathcal{R} \zeta)+g(-i r, \mathcal{R} \zeta) \leq g(i r, \zeta)+g(-i r, \zeta)
$$

Now (2.11), (2.15), (2.16) imply Proposition 1.

Proof of Proposition 2: Let $z \in \tilde{K}_{\varphi}$. Assume first that $z \in K \cap H$. Then $z \in K$. Moreover, if $\zeta \in K \cap \mathcal{R} H$, we have $g(z, \mathcal{R} \zeta) \geq g(z, \zeta)$. Hence (2.14) yields

$$
v(z) \geq \int_{K} g(z, \zeta) d \mu(\zeta)=u(z)=1 .
$$

Finally suppose that $z \in \mathcal{R}(K \cap \mathcal{R} H)$. In this case $\mathcal{R} z \in K$. If $\zeta \in K \cap H$, we have $g(z, \zeta) \geq g(z, \mathcal{R} \zeta)$. Hence (2.14) and the symmetry of the unit disk (with respect to the line $l(\varphi)$ ) give

$$
v(z) \geq \int_{K} g(z, \mathcal{R} \zeta) d \mu(\zeta)=\int_{K} g(\mathcal{R} z, \zeta) d \mu(\zeta)=u(\mathcal{R} z)=1
$$

So Proposition 2 and the lemma are proved.

Lemma 2.6. Let $0 \leq r<1,0<\theta<\frac{\pi}{2}$ and $\psi=\frac{\pi}{4}+\frac{\theta}{2}$. For $\zeta \in$ $\mathcal{R}_{\psi} H_{\psi} \cap\{z \in \mathbb{D}: \Re z \geq 0, \Im z \geq 0\}$, we have

$$
g\left(-i r, \mathcal{R}_{\psi} \zeta\right) \leq g\left(r e^{-i \theta}, \zeta\right)
$$

Proof. Let arg denote the principal branch of the argument. By Lemma 2.2 , it suffices to show that

$$
\arg \left(\mathcal{R}_{\psi} \zeta\right)-\arg (-i r) \geq \arg \zeta-\arg \left(r e^{-i \theta}\right)
$$

Simple calculations show that (2.20) is equivalent to $\arg \zeta \leq \pi / 2$ which is true. So the lemma is proved. 
Lemma 2.7. Let $0 \leq r<1,0<\theta<\frac{\pi}{2}$ and $\psi=\frac{\pi}{4}+\frac{\theta}{2}$. Also let $K$ be a compact set in $\overline{\mathbb{D}} \cap\{z: \Re z \geq 0\}$. Then

$$
\omega\left(r e^{i \theta}, K\right)+\omega\left(r e^{-i \theta}, K\right) \geq \omega\left(i r, \tilde{K}_{\psi}\right)+\omega\left(-i r, \tilde{K}_{\psi}\right) .
$$

Proof. The proof is very similar to the proof of Lemma 2.5. So we only sketch its main steps. We define the superharmonic function $u$ and construct the function $v$ as in the proof of Lemma 2.5. Then we have to prove two propositions. The second proposition and its proof are exactly the same as in Lemma 2.5. The first proposition is that

$$
v(i r)+v(-i r) \leq u\left(r e^{i \theta}\right)+u\left(r e^{-i \theta}\right) .
$$

To prove (2.22) we work as in the proof of Proposition 1 in Lemma 2.5. The only difference is that instead of (2.16) we need the inequality

$$
g\left(i r, \mathcal{R}_{\psi} \zeta\right)+g\left(-i r, \mathcal{R}_{\psi} \zeta\right) \leq g\left(r e^{i \theta}, \zeta\right)+g\left(r e^{-i \theta}, \zeta\right)
$$

for $\zeta \in K \cap \mathcal{R}_{\psi} H_{\psi}$. Since the points $i r, r e^{i \theta}$ and $\zeta, \mathcal{R}_{\psi} \zeta$ are symmetric in $l(\psi)$, we have

$$
g\left(i r, \mathcal{R}_{\psi} \zeta\right)=g\left(r e^{i \theta}, \zeta\right)
$$

Also, by Lemma 2.6, we have

$$
g\left(-i r, \mathcal{R}_{\psi} \zeta\right) \leq g\left(r e^{-i \theta}, \zeta\right), \zeta \in K \cap \mathcal{R}_{\psi} H_{\psi}
$$

Now (2.24) and (2.25) imply (2.23) and the lemma is proved.

\section{Proof of Theorem 1.1.}

Let $r, \theta, K$ be as in the statement of the theorem. By applying Lemma 2.4 first with $\varphi=\pi$ and (2.8) and then with $\phi=\pi / 2$ and (2.7), we may 
assume that $K \subset\{z \in \overline{\mathbb{D}}: \Re z \geq 0, \Im z \geq 0\}$. Suppose that $\theta=\pi / 2$. We apply Lemma 2.5 successively with $\varphi=\pi / 4, \pi / 8, \pi / 16, \ldots$. In the limit we obtain (1.2). So the theorem is proved for $\theta=\pi / 2$. Assume next that $\pi / 2<\theta \leq \pi$ and let $\mathbb{D}_{-}=\{z \in \mathbb{D}: \Re z<0\}$. By Lemma 2.1 and symmetry in the real axis,

$$
\begin{aligned}
& \omega\left(r e^{i \theta}, K\right)+\omega\left(r e^{-i \theta}, K\right) \\
= & \int_{[-i, i]} \omega(\zeta, K)\left[\omega\left(r e^{i \theta}, d \zeta, \mathbb{D}_{-}\right)+\omega\left(r e^{-i \theta}, d \zeta, \mathbb{D}_{-}\right)\right] \\
= & \int_{[0, i]}[\omega(\zeta, K)+\omega(\bar{\zeta}, K)]\left[\omega\left(r e^{i \theta}, d \zeta, \mathbb{D}_{-}\right)+\omega\left(r e^{-i \theta}, d \zeta, \mathbb{D}_{-}\right)\right] .
\end{aligned}
$$

Now by the first part of this proof (for $\theta=\pi / 2$ ), if $\zeta \in(0, i)$,

$$
\omega(\zeta, K)+\omega(\bar{\zeta}, K) \geq \omega\left(\zeta, K^{*}\right)+\omega\left(\bar{\zeta}, K^{*}\right) .
$$

By (2.26) and (2.27) and Lemma 2.1, we obtain (1.2), and the theorem is proved.

\section{Proof of Theorem 1.2.}

Let $r, \theta, K$ be as in the statement of the theorem. By applying Lemma 2.4 with $\varphi=\pi$ we may assume that $K \subset\{z \in \overline{\mathbb{D}}: \Re z \geq 0, \Im z \geq 0\}$. Let $\psi=\pi / 4+\theta / 2$. We apply Lemma 2.7 and obtain

$$
\omega\left(r e^{i \theta}, K\right)+\omega\left(r e^{-i \theta}, K\right) \geq \omega\left(i r, \tilde{K}_{\psi}\right)+\omega\left(-i r, \tilde{K}_{\psi}\right) .
$$

Applying again Lemma 2.4 with $\varphi=\pi$ we may assume that the set

$K_{\psi}$ lies in $\{z \in \overline{\mathbb{D}}: \Re z \geq 0, \Im z \geq 0\}$. Finally we apply Lemma 2.5 successively with $\varphi=\pi / 4, \pi / 8, \pi / 16, \ldots$ and in the limit we obtain (1.3). 


\section{REFERENCES}

1. D.Betsakos, An extension of the Beurling-Nevanlinna projection theorem. Computational Methods in Function Theory (CMFT'97). N.Papamichael, St.Ruscheweyh and E.Saff (Eds.), pp. 87-90. World Scientific, 1999.

2. D.Betsakos, Geometric theorems and problems for harmonic measure. Rocky Mountain J. Math. 31 (2001), 773-795.

3. J.L.Fernández, J.Heinonen, O.Martio, Quasilines and conformal mappings. J. d' Analyse Math. 52 (1989), 117-132.

4. B.Øksendal, Projection estimates for harmonic measure. Ark. Mat. 21 (1983), 191-203.

5. S.C.Port, C.J.Stone, Brownian Motion and Classical Potential Theory. Academic Press 1978.

6. T.Ransford, Potential Theory in the Complex Plane. Cambridge University Press 1995.

Department of Mathematics, Aristotle University of Thessaloniki, GR-54124 Thessaloniki, Greece

E-mail address: betsakos@auth.gr 